\title{
Fast Magnetization Switching in Amorphous Microwires
}

\author{
A. Zhukov ${ }^{a, b, *}$, M. Ipatov ${ }^{a}$, J.M. Blanco $^{c}$, A. ChizhiK $^{a}$, A. TAlaAT $^{a}$, V. Zhukova $^{a}$ \\ ${ }^{a}$ Dpto. Fisica de Materiales, Facultad de Química, UPV/EHU, 1072, 20018 San Sebastian, Spain \\ ${ }^{b}$ IKERBASQUE, Basque Foundation for Science, 48011 Bilbao, Spain \\ ${ }^{c}$ Dpto. de Física Aplicada, EUPDS, UPV/EHU, 20018, San Sebastian, Spain
}

\begin{abstract}
We studied the magnetization switching in magnetically bistable amorphous ferromagnetic microwires. We observed quite fast domain wall propagation along the microwires and a correlation between the magnetoelastic anisotropy, distribution of the local nucleation field along the length of microwire and the domain wall dynamics. We observed that both DW velocity and the range of fields, limiting single DW dynamics, can be manipulated by internal or applied stresses and by annealing. We also observed that under certain conditions a controllable domain wall (DW) collision can be realized in different parts of the wire, and that it is possible to manipulate the DW dynamics in a field-driven regime.
\end{abstract}

DOI: $10.12693 /$ APhysPolA.126.7

PACS: 75.60.Ej, 75.60.Jk

\section{Introduction}

Studies of fast and controllable domain wall (DW) propagation in thin magnetic wires attracted considerable attention due to promising applications in microand nanotechnology [1, 2]. From the point of view of applications the speed of controllable DW propagation is one of the most relevant parameters [2].

In the case of cylindrical microwires, exhibiting spontaneous magnetic bistability, the DW speed above $1 \mathrm{~km} / \mathrm{s}$ has been reported [2-4]. Therefore studies of DW dynamics in amorphous glass-coated microwires are quite important for understanding of the origin of fast DW propagation and ways to enhance the DW velocity in other materials.

Last attempts to study the micromagnetic origin of the head-to-head DW in microwires showed that this DW is rather thick and has complex structure [3, 4]. The characteristic width of the head-to-head DW, $\delta$, depends on many factors, such as applied magnetic field, $H$, magnetic anisotropy constant, $K$, and the wire diameter, $d[4]$.

The Taylor-Ulitovky method involves simultaneous rapid quenching of metallic nucleus inside the glass coating. Considerable difference of the thermal expansion coefficients of the glass and the metal, results in appearance of considerable internal stresses $[5,6]$. Both the applied and the internal stresses considerably affect soft magnetic properties of amorphous materials [7]. Consequently, in the absence of magnetocrystalline anisotropy the magnetoelastic anisotropy is the main factor, determining the magnetic properties of glass-coated microwires and the DW propagation $[8,9]$.

One the other hand, fabrication technique involves metallurgical processes at elevated temperatures under

*corresponding author; e-mail: arkadi.joukov@ehu.es the electromagnetic field of inductor affecting the alloy ingot [10]. Recently we showed that the microwires's inhomogeneities sufficiently affect the remagnetization process of magnetically bistable microwires, limiting single DW propagation regime as well as the domain wall propagation velocity, $v[11,12]$. The origin of the defects is still unclear.

Various attempts to manipulate DW motion through the pinning at artificially created defects in thin magnetic wires have been reported $[13,14]$. It was reported recently on the successful braking and trapping of DWs in microwires under the influence of an additional antiparallel local magnetic field [15] and on collisions between two moving DWs in a magnetic nanowire, driven by an externally applied field [16]. In the latter case the DW can be controlled to allow for both domain wall annihilation and preservation during the collisions as long as the wire remains thin.

In this paper, we demonstrate the possibility to manipulate the DW dynamics in magnetic microwires by tailoring the magnetoelastic anisotropy, controlling the defects and by controlled DW collision.

\section{Experimental method}

We prepared a few amorphous Fe-Co-Ni based glasscoated microwires with positive magnetostriction constant, with different ratio of metallic nucleus diameter and the total diameter, $D$, i.e. with different ratios $\rho=d / D$, and with different composition of metallic nucleus [10]. This allowed us to control residual stresses, since the strength of internal stresses is determined by ratio $\rho[10]$, and to change the magnetostriction constant $[17,18]$.

The technique allowing measurement of the DW velocity in thin wires is well described elsewhere $[2,12]$. In our experiment we placed one end of the sample outside of the magnetization solenoid, in order to always activate the DW propagation from the other end of the wire. We have used three pick-up coils, mounted along the length 
of the wire. The propagating DW induces electromotive force (emf) in the coils, as described in Ref. 12. These sharp emf peaks are picked up at an oscilloscope upon passing of the propagating domain wall.

Then, DW velocity is estimated as

$$
v=\frac{l}{\Delta t}
$$

where $l$ is the distance between pick-up coils and $\Delta t$ is the time difference between the maximum in the induced emf.

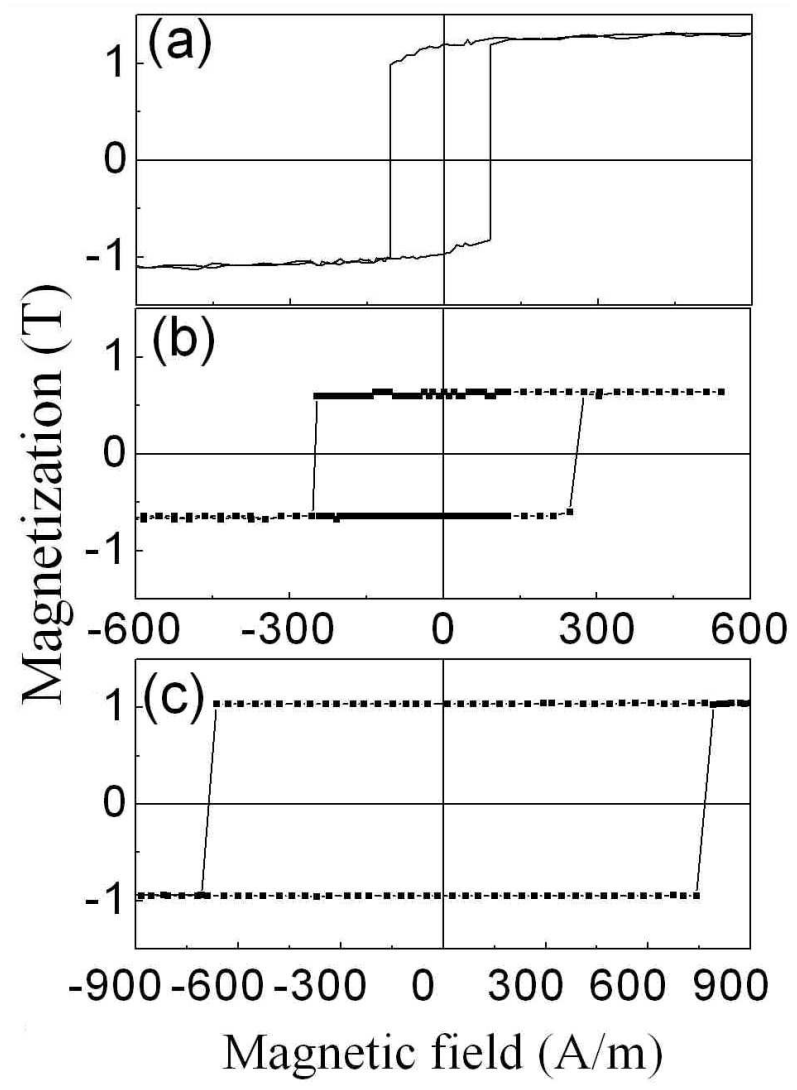

Fig. 1. Hysteresis loops of $\mathrm{Fe}_{70} \mathrm{~B}_{15} \mathrm{Si}_{10} \mathrm{C}_{5}$ amorphous microwires with different $\rho$-ratios: $\rho=0.63, d=$ $15 \mu \mathrm{m}(\mathrm{a}) ; \rho=0.48, d=10.8 \mu \mathrm{m}$ (b) and of $\mathrm{Fe}_{72.75} \mathrm{Co}_{2.25} \mathrm{~B}_{15} \mathrm{Si}_{10}$ microwire with $\rho=0.14, d=$ $1.4 \mu \mathrm{m}(\mathrm{c})$.

We used simple measurement method based on the classical Sixtus-Tonks-like experiments [12], allowing application of tensile stresses when measuring the DW dynamics in microwires.

For determination of the nucleation field profile we used the method described in [11].

Hysteresis loops have been measured using vibrating sample magnetometer (VSM) and fluxmetric technique described elsewhere [10].

\section{Experimental results and discussion}

The magnetoelastic energy, $K_{m e}$, is given by

$$
K_{m e} \approx 3 / 2 \lambda_{s} \sigma \text {, }
$$

where $\sigma=\sigma_{i}+\sigma_{a}$ - total stress, $\sigma_{i}$ - the internal stresses, $\sigma_{a}$ - applied stresses and $\lambda_{s}-$ magnetostriction constant [10].

Hysteresis loops of Fe-rich microwires present considerable dependence on the $\rho$-ratio (Fig. 1). To confirm magnetoelastic origin of the observed changes of hysteresis we have measured the effect of applied stresses, $\sigma_{a}$, on switching field, $H_{s}$, defined as the field at which the large Barkhausen jump starts. At low magnetic field amplitudes and frequencies the $H_{s}$ is almost the same as coercivity, $H c$. However the switching field $H_{s}$ is usually almost independent on magnetic field amplitude, $H_{0}$ and frequency, $f$ [19]. The difference between $H_{s}$ and $H_{c}$ was attributed to the DW dynamics, i.e. the time of domainwall propagation through the entire wire [19]. Therefore use of $H_{s}$ for studying of stress dependence of hysteresis loops is more rigorous.

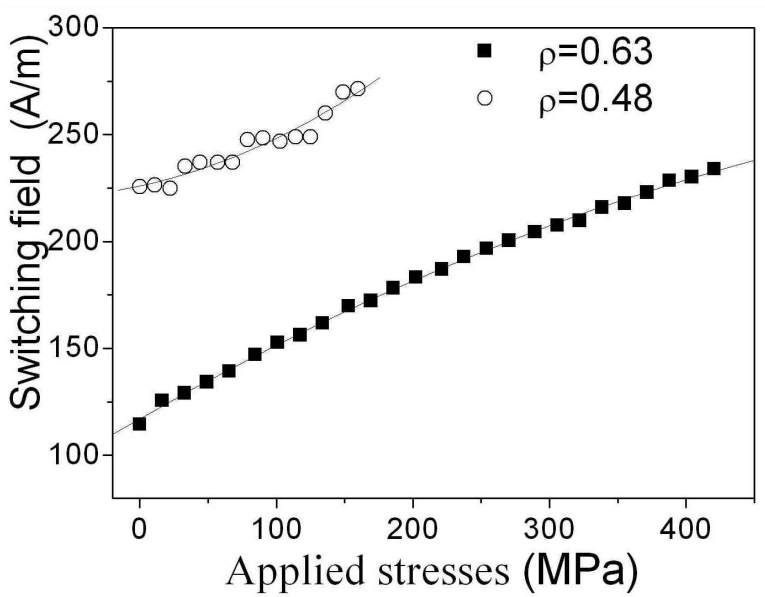

Fig. 2. Effect of applied stresses on switching field of $\mathrm{Fe}_{70} \mathrm{~B}_{15} \mathrm{Si}_{10} \mathrm{C}_{5}$ amorphous microwires with different $\rho$ ratios: $\rho=0.63, d=15 \mu \mathrm{m}$ and $\rho=0.48, d=10.8 \mu \mathrm{m}$.

We observed considerable and monotonic growth of $H_{s}$ with $\sigma_{a}$ (Fig. 2). Consequently from comparison of Figs. 1 and 2 we can conclude that the internal stresses in glass-coated microwires have mostly tensile character (as also shown in previous papers $[5,6]$ ), and that the strength of internal stresses increases with decreasing $\rho$ ratio. It is worth mentioning, that the observed stress dependence of $H_{s}$ is similar to one, previously observed for microwires with similar compositions [20]. However, the presented here $H_{s}\left(\sigma_{a}\right)$ dependences have been obtained considering stress distribution between the metallic nucleus and glass coating, i.e. considering composite character of microwires, as has been described in Ref. 21 .

Figure 3 shows the distribution of the local nucleation fields $H_{n}$ along the sample length, $L$, measured in $\mathrm{Fe}_{74} \mathrm{~B}_{13} \mathrm{Si}_{11} \mathrm{C}_{2}$ microwire, as described above. As we can observe from Fig. 3, the nucleation field near wire ends is considerably smaller. This difference between the nucleation field near the sample ends and the body of the microwire is responsible for the onset of large Barkhausen 


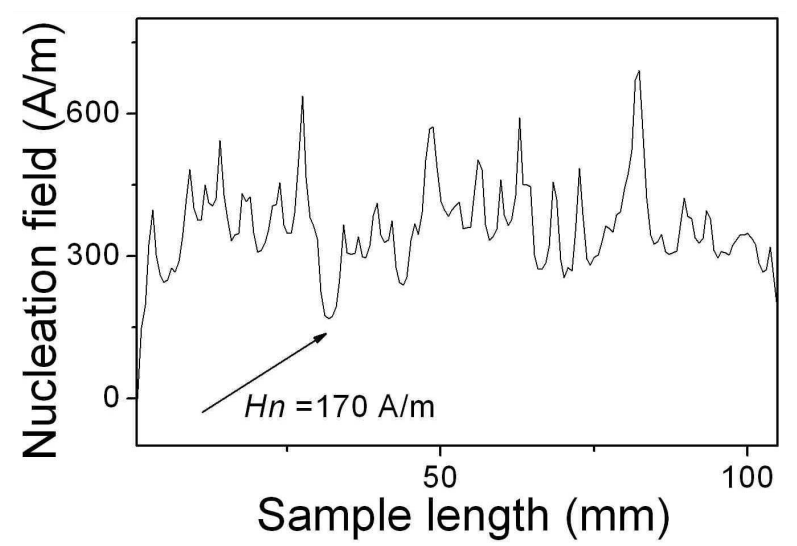

Fig. 3. Distribution of the local nucleation fields, measured in amorphous $\mathrm{Fe}_{74} \mathrm{~B}_{13} \mathrm{Si}_{11} \mathrm{C}_{2}$ microwire $(d=$ $19.4 \mu \mathrm{m}, \rho=0.73)$.

jump, in turn responsible for the rectangular character of hysteresis loop. Additionally this difference determines the limits of single DW propagation regime, as reported recently by us [8]. On the other hand, the observed deep pits on $H_{n}(L)$ curves must be attributed to the positions of localized defects existing within the microwire, as also has been previously reported $[11,12]$.

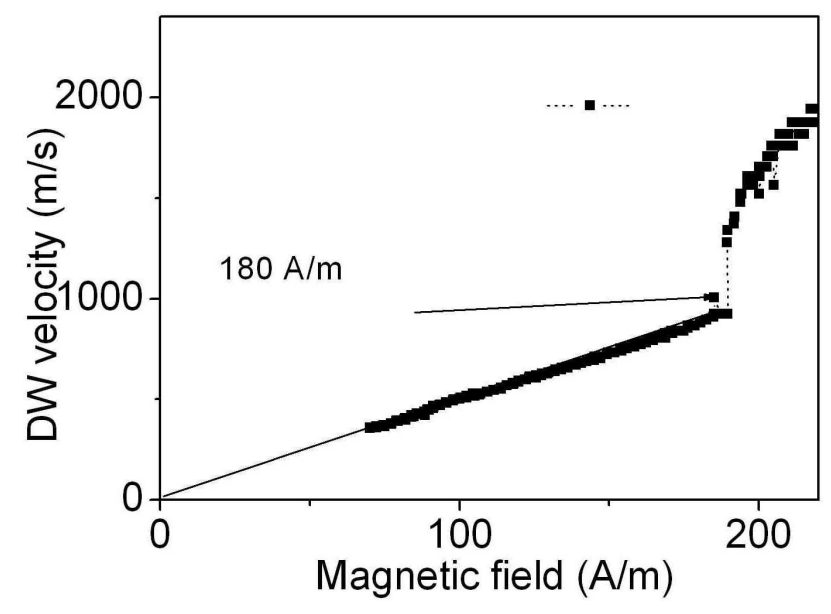

Fig. 4. $v(H)$ dependence measured in amorphous $\mathrm{Fe}_{74} \mathrm{~B}_{13} \mathrm{Si}_{11} \mathrm{C}_{2}$ microwire $(d=19.4 \mu \mathrm{m} ; \rho=0.73)$.

In Fig. 4 we observe the dependence of DW velocity, $v$, on applied magnetic field, $H$, measured in the same sample.

The abrupt jump on the dependence of DW velocity on magnetic field must be attributed to the nucleation of additional DW on sample inhomogeneities, since the field of the jump correlates well with the minimum nucleation field observed in Fig. 3.

Below the jump on $v(H)$ dependence we observed a linear increase of DW velocity, $v$, with $H$, as previously observed in most of cases for Fe-rich microwires [12]. The origin of observed inhomogeneities is unclear and at least partially can be related with the distribution of internal stresses along the microwire. A conventional way of stress release is the heat treatment. Consequently we performed sample annealing and measured $v(H)$ dependences in the as-prepared microwire and the microwire after heat treatments (see Fig. 5). We observed, that annealing of the $\mathrm{Fe}_{74} \mathrm{~B}_{13} \mathrm{Si}_{11} \mathrm{C}_{2}$ microwire, performed at $300{ }^{\circ} \mathrm{C}$ for $60 \mathrm{~min}$, considerably affects $v(H)$ dependence: DW velocity measured at the same magnetic field considerably increases, the range of fields where linear $v(H)$ dependence takes place is extended and DW mobility, $S$, increased from 4.02 to $4.65 \mathrm{~m}^{2} \mathrm{~A}^{-1} \mathrm{~s}^{-1}$ (Fig. 5).

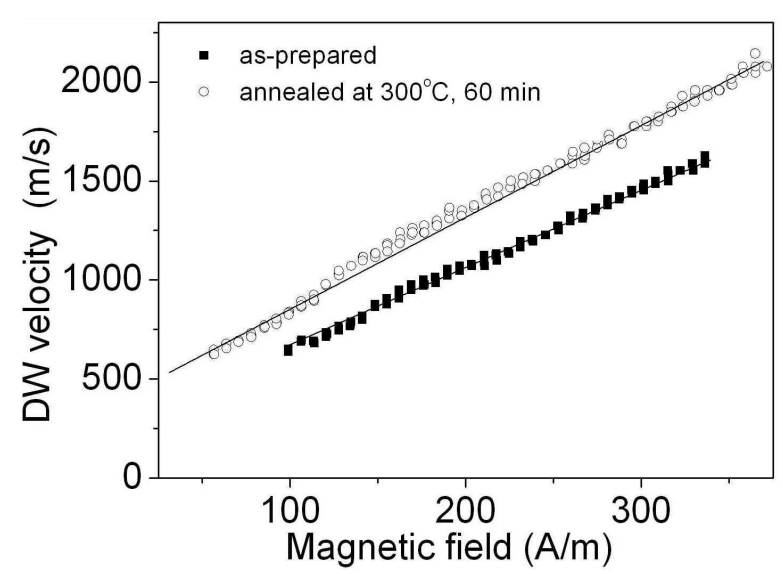

Fig. 5. Effect of annealing on the $v(H)$ dependence of $\mathrm{Fe}_{74} \mathrm{~B}_{13} \mathrm{Si}_{11} \mathrm{C}_{2}$ microwire $(d=14.6 \mu \mathrm{m}, \rho=0.55)$.

The observed influence of sample annealing on DW dynamics can be interpreted, considering that the observed inhomogeneities, at least partially, have magnetoelastic origin. In this way, sample annealing partially releases the stresses, related with simultaneous solidification of metallic nucleus inside the glass coating during the microwire casting.

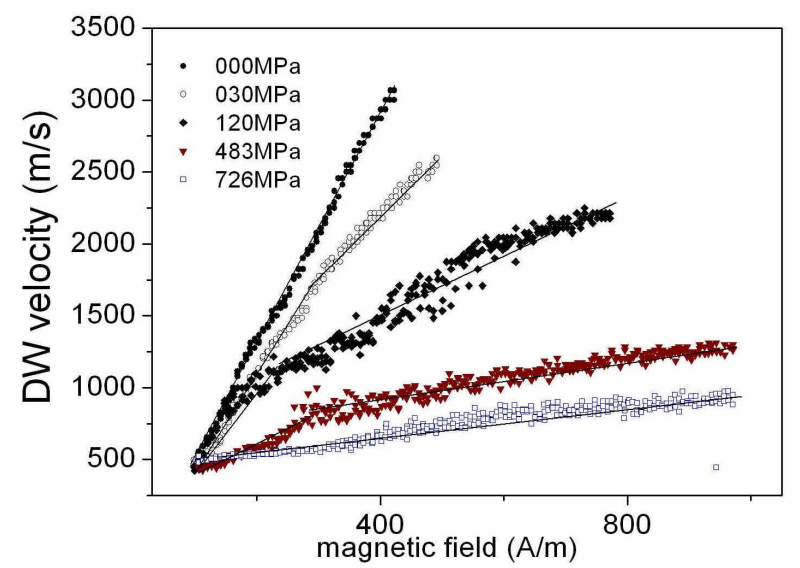

Fig. 6. $v(H)$ dependences for $\mathrm{Fe}_{74} \mathrm{~B}_{13} \mathrm{Si}_{11} \mathrm{C}_{2}$ microwire $(d \approx 14.6 \mu \mathrm{m}, D \approx 21.8 \mu \mathrm{m}, \rho \approx 0.55)$, measured under application of stress, $\sigma_{a}$. 
Applied stress considerably affected the $v(H)$ dependence (Fig. 6). This confirms that the magnetoelastic anisotropy considerably affects DW dynamics and overall remagnetization process of glass-coated ferromagnetic microwires.

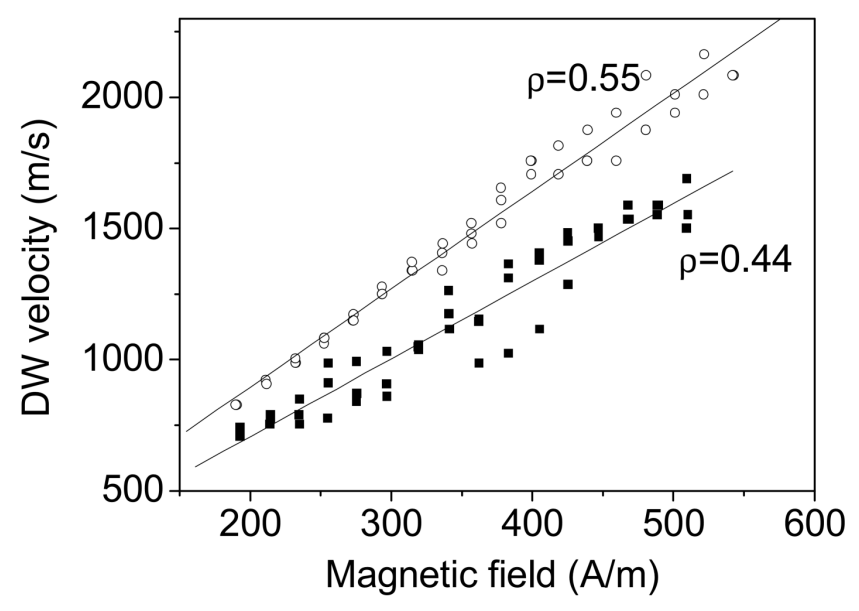

Fig. 7. $v(H)$ dependences for the $\mathrm{Fe}_{55} \mathrm{Co}_{23} \mathrm{~B}_{11.8} \mathrm{Si}_{10.1}$ microwires with different ratios $\rho$.

In order to evaluate the effect of $\rho$-ratio, i.e. the effect of residual stresses on DW dynamics, we performed measurements of $v(H)$ dependences in the microwires with the same composition, but with different $\rho$-ratios. Dependences of DW velocity on applied field for $\mathrm{Fe}_{55} \mathrm{Co}_{23} \mathrm{~B}_{11.8} \mathrm{Si}_{10.1}$ microwires with different ratios are shown in Fig. 7 .

Consequently, by manipulation of the magnetoelastic energy through application of tensile stress, by changing the magnetostriction constant and the internal stresses in studied microwires we have significantly affected the DW dynamics in magnetically bistable microwires.

The application of the bias magnetic field, $H_{b}$ at an angle of $\Theta \sim 85^{\circ}$ with respect to the wire axis can activate the propagation of the $\mathrm{DW}$ from the end of the wire located outside the solenoid.

As can be appreciated from Figs. 8(a, b), by changing value of $H_{b}$ (and consequently the axial $H_{b}$ projection) we can activate in a controllable way the DW propagation from the opposite end of the sample and induce a DW collision at various locations in the sample. If this collision takes place in the vicinity of a pick-up coil we will observe an increase of the voltage peak amplitude. Consequently we are able to engineer the DW collisions between two moving DWs in different places of the magnetic microwire by controlling the applied bias field.

\section{Conclusions}

We have experimentally observed that manipulating the magnetoelastic energy through application of tensile stress, changing the magnetostriction constant and internal stresses in studied microwires we significantly affected the domain wall dynamics in magnetically bistable

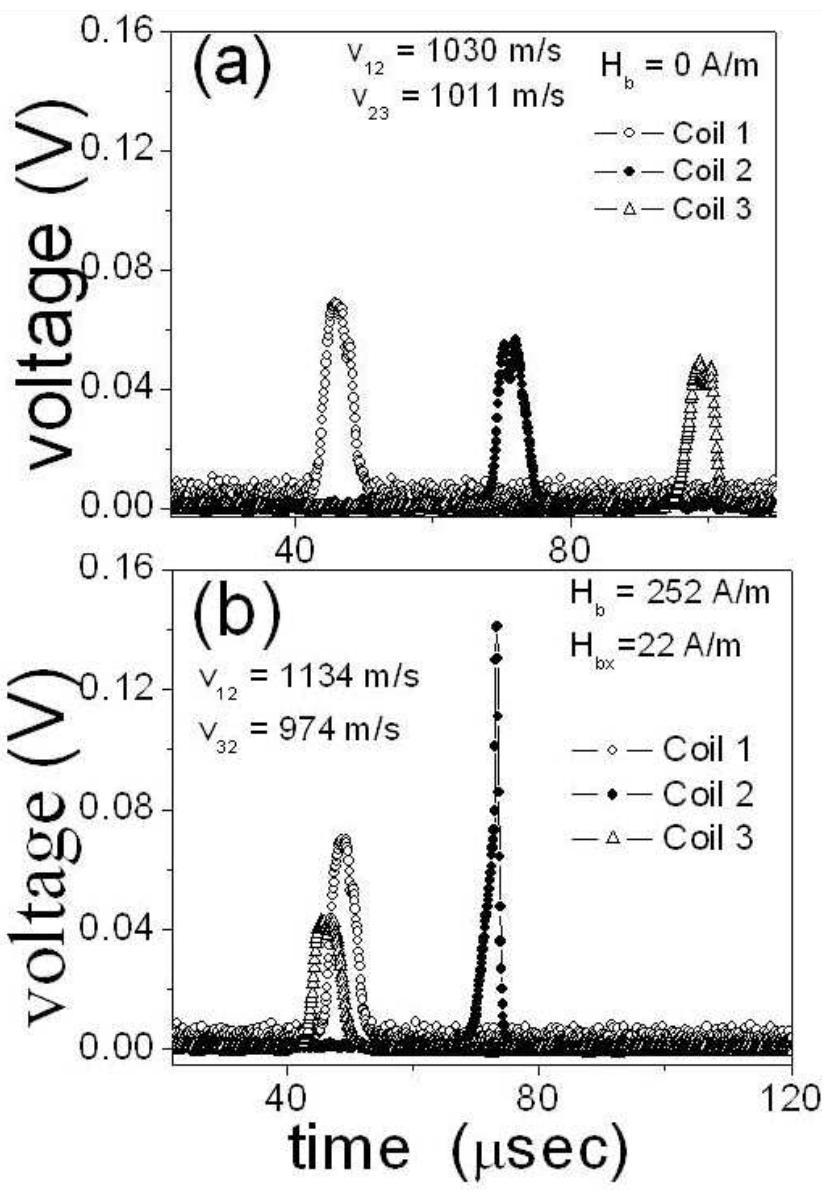

Fig. 8. Voltage peaks, induced by the propagating DW in the 3 pick-up coils in $\mathrm{Fe}_{74} \mathrm{~B}_{13} \mathrm{Si}_{11} \mathrm{C}_{2}(d \approx 14.6 \mu \mathrm{m}$, $D \approx 21.8 \mu \mathrm{m}, \rho \approx 0.67)$ microwires measured at $H=$ $140 \mathrm{~A} / \mathrm{m}$ with different values of bias magnetic field, $\mathrm{H}_{b}$, and its axial projection $\mathrm{H}_{b x}$.

microwires. Strong correlation between the type of the $v(H)$ dependence and the distribution of the local nucleation fields has been observed.

Under certain experimental conditions it is possible to manipulate the DW dynamics in a magnetic wire in a field-driven regime and observe controllable DW collisions. This controllable DW collision can be realized in different parts of the wire. Such DW collisions can be used to release the pinned domain walls using weak external fields.

\section{Acknowledgments}

This work was supported by EU ERA-NET programme under project "SoMaMicSens" (MANUNET2010-Basque-3), by Spanish MICINN under project MAT2010-18914 and by the Basque Government under Saiotek-12 MEMFOMAG project (S-PE12UN139). A. Zh. and V.Zh. wish to acknowledge support of the Basque Government under Program of Mobility of the Investigating Personnel (grants MV-2013-2-22 and MV- 
2013-2-23). Technical and human support provided by SGIker (UPV/EHU, MICINN, GV/EJ, ERDF and ESF) is gratefully acknowledged.

\section{References}

[1] M. Hayashi, L. Thomas, Ch. Rettner, R. Moriya, X. Jiang, S. Parkin, Phys.Rev. Lett. 97, 207205 (2006).

[2] R. Varga, A. Zhukov, V. Zhukova, J.M. Blanco, J. Gonzalez, Phys. Rev. B 76, 132406 (2007).

[3] P.A. Ekstrom, A. Zhukov, J. Phys. D: Appl. Phys. 43, 205001 (2010).

[4] S.A. Gudoshnikov, Yu.B. Grebenshchikov, B.Ya. Ljubimov, P.S. Palvanov, N.A. Usov, M. Ipatov, A. Zhukov, J. Gonzalez, Phys. Status Solidi A 206, 613 (2009).

[5] A.S. Antonov, V.T. Borisov, O.V. Borisov, A.F. Prokoshin, N.A. Usov, J. Phys. D: Appl. Phys. 33, 1161 (2000).

[6] H. Chiriac, T.-A. Ovari, A. Zhukov, J. Magn. Magn. Mater. 254-255, 469 (2003).

[7] J.M. Blanco, A. Zhukov, J. Gonzalez, J. Appl. Phys. 87, 4813 (2000).

[8] A. Zhukov, J.M. Blanco, M. Ipatov, A. Chizhik, V. Zhukova, Nanoscale Research Letters 7, 223 (2012).

[9] K. Richter, R. Varga, A. Zhukov, J. Phys. C: Condens. Matter 24, 296003 (2012).
[10] V. Zhukova, M. Ipatov, A Zhukov, Sensors 9, 9216 (2009).

[11] M. Ipatov, N.A. Usov, A. Zhukov, J. González, Physica B 403, 379 (2008).

[12] M. Ipatov, V. Zhukova, A.K. Zvezdin, A. Zhukov, J. Appl. Phys. 106, 103902 (2009).

[13] S.-H. Huang, Ch.-H. Lai, Appl. Phys. Lett. 95, 032505 (2009).

[14] C.C. Faulkner, D.A. Allwood, R.P. Cowburn, J. Appl. Phys. 103, 073914 (2008).

[15] M. Vázquez, G.A. Basheed, G. Infante, R.P. Del Real, Phys.Rev. Lett. 108, 037201 (2012).

[16] A. Kunz, Appl. Phys. Lett. 94, 132502 (2009).

[17] A. Zhukov, V. Zhukova, J.M. Blanco, A.F.Cobeño, M. Vazquez, J. Gonzalez, J. Magn Magn. Mater 258-259, 151 (2003).

[18] Y. Konno, K. Mohri, IEEE Trans. Magn. 25, 3623 (1989).

[19] A. Zhukov, M. Vázquez, J. Velázquez, C. Garcia, R. Valenzuela, B. Ponomarev, J. Mat. Sci. Eng. A 226-228, 753 (1997).

[20] P. Aragoneses, J.M. Blanco, L. Dominguez, J. González, A. Zhukov, M. Vázquez, J. Phys. D: Appl. Phys. 31, 3040 (1998).

[21] V. Zhukova, V.S. Larin, A. Zhukov, J. Appl. Phys. 94, 1115 (2003). 